\title{
Oscillations in the PMS $\delta$ Scuti star V346 Ori ${ }^{\star}$
}

\author{
F. J. G. Pinheiro ${ }^{1}$, D. F. M. Folha ${ }^{1}$, M. Marconi ${ }^{2}$, V. Ripepi ${ }^{2}$, F. Palla ${ }^{3}$, M. J. P. F. G. Monteiro ${ }^{1,4}$, and S. Bernabei ${ }^{5,6}$ \\ ${ }^{1}$ Centro de Astrofísica da Universidade do Porto, Rua das Estrelas, 4150-762 Porto, Portugal \\ 2 INAF-Osservatorio Astronomico di Capodimonte, Via Moiariello 16, 80131 Napoli, Italy \\ 3 INAF-Osservatorio Astrofisico di Arcetri, Largo E. Fermi 5, 50125 Firenze, Italy \\ ${ }^{4}$ Departamento de Matemática Aplicada, Faculdade de Ciências da Universidade do Porto, Portugal \\ 5 INAF-Osservatorio Astronomico di Bologna, Via Ranzani 1, 40127 Bologna, Italy \\ ${ }^{6}$ Departamento de Astrofísica, Universidad de La Laguna, Avda. Astrofísico F. Sánchez s/n, Campus de Anchieta, \\ 38206 La Laguna, Spain
}

Received 16 September 2002 / Accepted 27 November 2002

\begin{abstract}
We report the discovery of periodicities in the light curve of the Pre-Main Sequence (PMS) Herbig Ae star V346 Ori. We interpret variations in the light curve of the star as the superposition of at least two periodic signals that result from $\delta$ Scuti-type stellar oscillations. The computation of linear non-adiabatic radial pulsation models for PMS stars reproduces these periods for a $1.55 M_{\odot}$ star with $T_{\text {eff }}=7410 \mathrm{~K}$ and $\log L / L_{\odot}=0.74$ pulsating in the fundamental and in the second overtone. If our assumption of radial oscillations is indeed correct, the star's location on the HR diagram places it at a distance significantly smaller than that of the Orion star forming region, hence raising questions regarding the evolutionary status of V346 Ori. Observations with better time sampling and spanning a longer period of time are needed if the latter question is to be addressed fully.
\end{abstract}

Key words. stars: evolution - stars: fundamental parameters - stars: oscillations - stars: pre-main sequence stars: variables: $\delta$ Sct - stars: individual: V346 Ori

\section{Introduction}

Pre-Main sequence (PMS) evolutionary tracks are of utmost importance to achieve a complete understanding of the star formation process. Such tracks allow the determination of both mass and age of individual young stars (eg. Hillenbrand 1997). These parameters are fundamental data not only to characterise the stars themselves but also to investigate how the initial mass function varies from region to region as a function of cloud parameters.

PMS evolutionary models are produced by different groups, each employing different treatments of convection and/or opacities and also using a different zero-point for the calculated ages. How is one to assess the accuracy of model predictions and, possibly, to choose that which better describes the evolution of stars during their contraction towards the main sequence?

A powerful test to models is to independently and accurately derive the mass of PMS objects and compare their position in the HR diagram with model predictions for the determined masses. Eclipsing binary systems provide the best

Send offprint requests to: D. F. M. Folha,

e-mail: dfmf@astro.up.pt

* Tables 1 and 2 are only available in electronic form at the CDS via anonymous ftp to cdsarc.u-strasbg.fr $(130.79 .128 .5)$ or via http://cdsweb.u-strasbg.fr/cgi-bin/qcat?J/A+A/399/271 estimates of stellar masses (Popper 1980; Palla \& Stahler 2001). Unfortunately, only a few such systems are known that are sufficiently young to provide a test to the PMS evolutionary tracks (Covino et al. 2001).

Marconi \& Palla (1998) investigated the pulsational properties of PMS stars within the mass range $1-4 M_{\odot}$, by means of non-linear calculations of radial modes and derived the location in the HR diagram of the instability strip for these stars. The identification of pulsation frequencies in these stars allows to constrain fundamental stellar parameters such as the mass, luminosity and effective temperature. This represents a powerful method to determine the stellar mass for stars that are not part of the very restricted group of spectroscopic binary systems. In this way, the number of PMS stars with accurately determined masses can be increased, allowing more stringent testing of the PMS evolutionary models.

Several Herbig Ae stars have been identified as pulsators. Particular attention has been given to HR 5999 (Kurtz \& Marang 1995; Kurtz \& Catala 2001) and V351 Ori (Marconi et al. 2000, 2001; Ripepi et al. A\&A, submitted). The potential of pulsations for the determination of stellar parameters is attested by Marconi et al. 2001, 2002). Depending on the accuracy, number and mode identification of discovered frequencies, single digit precisions (in percentage) can be achieved for the stellar mass and radius. Furthermore, for the latter two parameters, there is the additional advantage that their 


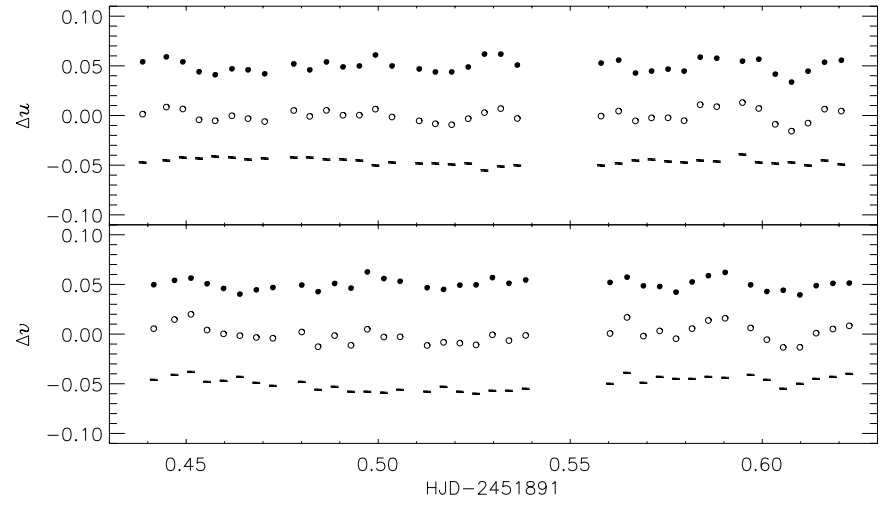

Fig. 1. Top panel: V346 Ori differential light curves of the data taken 12 December 2000, in the Strömgren $u$ band with respect to HD 35408 (filled circles) and to HD 35409 (open circles). The size of error bars is smaller than the circles size. The HD 35408 minus HD 35409 light curve is shown with error bars. Bottom panel: same as above for the Strömgren $v$ band.

determination from oscillations is independent of external factors, such as reddening.

V346 Ori (HD 287841) is classified as a Herbig Ae star (Thé et al. 1994) and given the estimates for its luminosity and effective temperature (van den Acker et al. 1998; Valenti et al. 2000) it is located near or inside the PMS instability strip. It is therefore a good candidate to display stellar oscillations. With the aim of increasing the number of known PMS pulsators we have searched for periodic variability in V346 Ori's light curve. In this paper we report our findings.

The paper is organised as follows: Sect. 2 describes the observations and the data reduction, in Sect. 3 we carry out the frequency analysis and in Sect. 4 we model the derived frequencies and discuss the results.

\section{Observations and data reduction}

The data presented here was taken on the nights of 12 December 2000 at the Isaac Newton Group (ING) $1.0 \mathrm{~m}$ Jacobus Kapteyn Telescope (JKT) in La Palma (Canary Islands, Spain) and 4 November 2001 at the $1.52 \mathrm{~m}$ "Cassini" telescope (Loiano, Italy).

The JKT was used with the JAG CCD camera equipped with a $1024 \times 1024$ pixels Tek CCD detector, providing a $5.5 \times$ 5.5 arcmin useful field-of-view (fov), and with a Strömgren filter set. The strategy was to observe target and comparison stars simultaneously by including them all on the fov and alternate the exposures between the Strömgren $u$ and $v$ filters, where the amplitude of oscillations are expected to be higher. Exposure times were 180 and $50 \mathrm{~s}$, in the $u$ and $v$ filters respectively, which added to the CCD read-out-time implied two consecutive data points in the same filter about every $7 \mathrm{~min}$. Observations started at HJD $=2451891.43865$ and had a duration of 4.366 hours. Two comparison stars were included on fov, namely HD 35408 and HD 35409.

On the "Cassini" telescope, the three channel photometer TTCP (see www.na.astro.it/ silvotti/TTCP.html for details on this instrument) was equipped with Johnson $U, B$

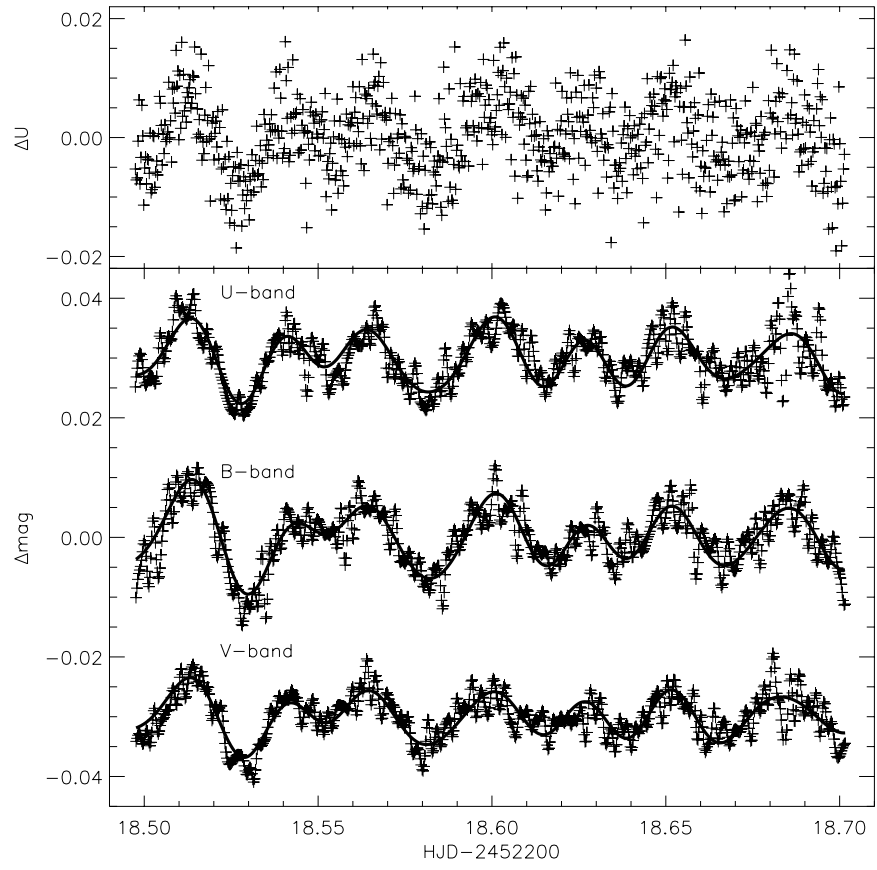

Fig. 2. V346 Ori light curves. Top panel: de-trended $U$-band data. Bottom panel: $U$-, $B$ - and $V$-band filtered data (see text). Solid lines: best fit of four frequencies (Sect. 3). The $U$ and $V$ data were shifted vertically for clarity.

and $V$ filters. The TTCP allows simultaneous observation of target star, comparison star and sky. We used an aperture of 17 arcsec for each channel. The exposure times were 15, 3 and $5 \mathrm{~s}$ in $U, B$ and $V$ respectively, thus, for a given filter, we obtained a differential photometry sampling rate of one data point about every 24 s. Observations started at HJD = 2452218.49745 and had the duration of 4.969 hours. The comparison star used was HD 35351 ( $B=9.07$ and spectral type A0), which is located $\sim 8.0$ arcmin away from V346 Ori.

The JKT data was reduced using standard data reduction procedures under IRAF, for bias subtraction and flatfield correction. Photometry was carried out with DAOPHOT, also under IRAF, using an aperture size of 8.25 arcsec radius. The TTCP data was reduced following the usual procedure: 1) normalisation of the response of each channel; 2) sky subtraction for variable and comparison star counts; 3) determination of differential magnitude variable-comparison star.

\section{Periodicities in the light curve}

To search for periodic variability in the V346 Ori light curve we used the Lomb Normalised Periodogram - LNP - (Lomb 1976; Scargle 1982), as implemented by IDL. Uncertainties in the value of detected frequencies were estimated from the full width at half maximum of the respective peaks in the LNP.

\subsection{Data set 1: December 2000}

Figure 1 shows the V346 Ori light curve as observed in 12 December 2000. In Table 1 we list the individual data points. 

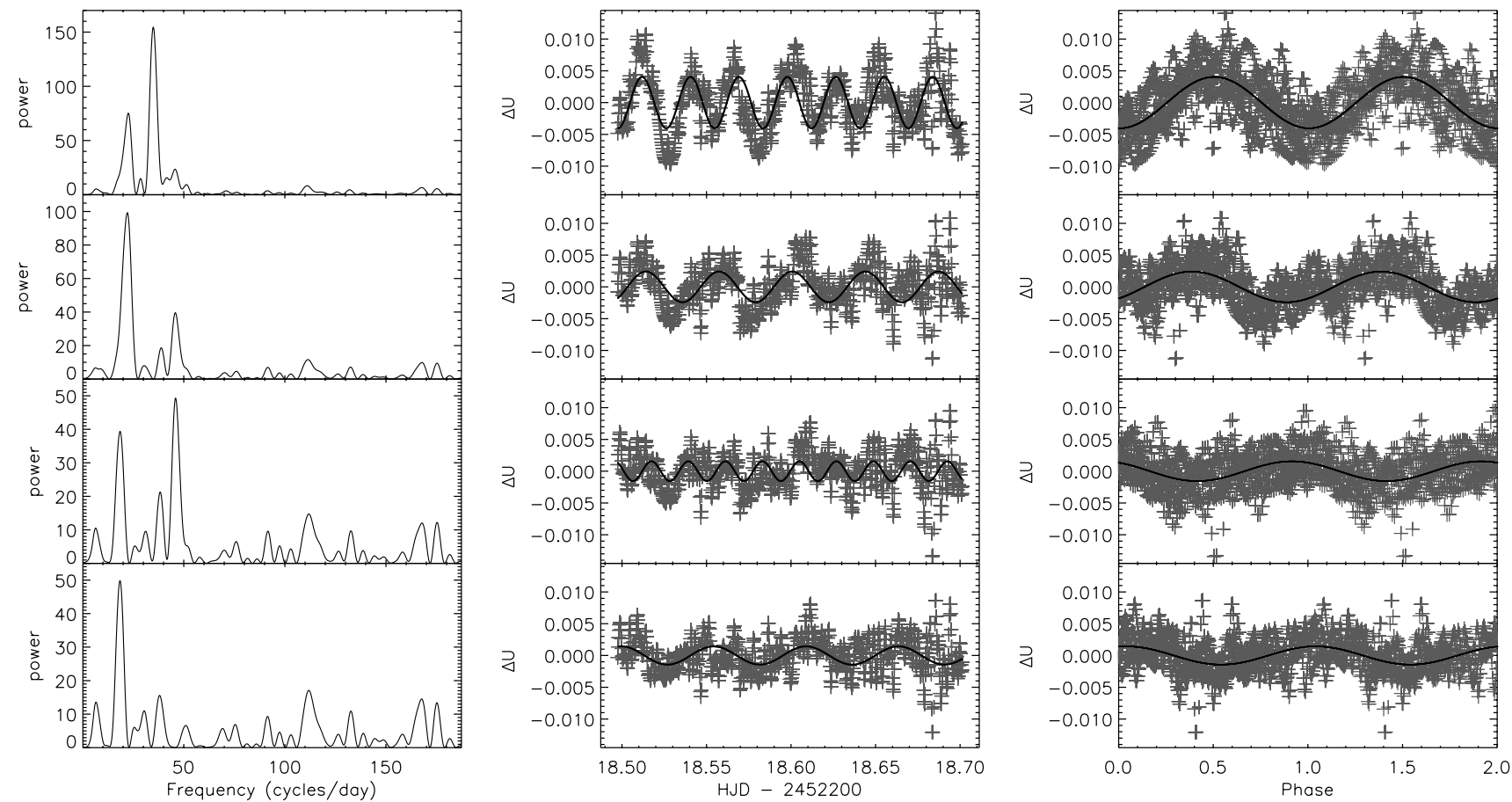

Fig. 3. Frequency analysis of the filtered data taken the 4th of November in the $U$ band. Top to bottom: light curve, light curve minus f1, light curve minus f1 and f2, light curve minus f1, f2 and f3. Left to right: Lomb Normalised Periodogram, light curve (or residual light curve) and phase diagram. The best fit to each frequency is overploted as a solid line in the middle and right hand side panels.

Table 3. Observed frequencies and associated amplitudes.

\begin{tabular}{cccccccc}
\hline \hline & \multicolumn{2}{c}{$U$-band } & \multicolumn{2}{c}{$B$-band } & \multicolumn{2}{c}{$V$-band } & Aver. freq. $\left(\mathrm{d}^{-1}\right)$ \\
& freq. $\left(\mathrm{d}^{-1}\right)$ & Amp. $(\mathrm{mmag})$ & freq. $\left(\mathrm{d}^{-1}\right)$ & Amp. $(\mathrm{mmag})$ & freq. $\left(\mathrm{d}^{-1}\right)$ & Amp. (mmag) & \\
\hline f1 & $34.9 \pm 2.2$ & $3.9 \pm 0.2$ & $35.3 \pm 2.1$ & $3.6 \pm 0.3$ & $35.4 \pm 2.2$ & $2.9 \pm 0.2$ & $35.2 \pm 1.3$ \\
f2 & $23.1 \pm 2.7$ & $2.3 \pm 0.2$ & $22.6 \pm 2.6$ & $4.3 \pm 0.3$ & $22.1 \pm 2.3$ & $2.8 \pm 0.2$ & $22.6 \pm 1.5$ \\
f3 & $45.7 \pm 2.3$ & $1.6 \pm 0.2$ & $45.9 \pm 2.2$ & $1.3 \pm 0.3$ & $45.4 \pm 2.1$ & $1.2 \pm 0.2$ & $45.7 \pm 1.3$ \\
f4 & $18.3 \pm 2.3$ & $1.5 \pm 0.2$ & $19.4 \pm 2.2$ & $2.1 \pm 0.3$ & $18.4 \pm 2.5$ & $1.4 \pm 0.2$ & $18.7 \pm 1.3$ \\
\hline
\end{tabular}

The uncertainty in each data point is 3 mmag and 2 mmag, respectively in the $u$ and $v$ differential light curves of V346 Ori.

The LNP analysis of the V346 Ori light curves yields two possible frequencies. In the $u$ band the two highest peaks in the LNP occur at frequencies $34.1 \pm 4.4 \mathrm{~d}^{-1}$ and $20.9 \pm 4.4 \mathrm{~d}^{-1}$, with false-alarm probabilities of 0.015 and 0.17 respectively. In the $v$ band the values are $35.2 \pm 4.9 \mathrm{~d}^{-1}$ and $22.0 \pm 4.4 \mathrm{~d}^{-1}$ for the frequencies and 0.02 and 0.18 for the false-alarm probabilities. Similar values are obtained if HD 35409 is used as comparison star for V346 Ori.

Figure 1 shows brightness variations in the comparison stars differential light curve, indicating that at least one of them may be itself variable. The LNP of the HD 35408 minus HD $35409 u$ and $v$ band light curves shows only a peak occuring near the 3 hours period $\left(8 \mathrm{~d}^{-1}\right)$, which is not significant given our total time sampling of 4.4 hours. Hence, the frequencies identified in the V346 Ori $u$ and $v$ light curves seem to result from the PMS star itself. We note, however, the relatively high false-alarm probabilities that result from this data set.

\subsection{Data set 2: November 2001}

The de-trended and zero averaged, 4 November $2001 U$-band light curve is shown in the top panel of Fig. 2. In Table 2 we list the individual data points. Uncertainties in the differential photometry are $3.5,2.5$ and $2.5 \mathrm{mmag}$, respectively in the $U$-, $B$ - and $V$-band. The error bars that result from such values are typically comparable with the symbol size, from which one quickly perceives the presence of very high frequency variations that are not due to the photon noise in the data, but instead result from scintillation noise caused by the very short integration times. To remove these high frequency fluctuations we applied a low pass filter, using a cut-off frequency of $490 \mathrm{~d}^{-1}$, i.e. high enough to avoid affecting the $\delta$ Scuti-type of periods being searched. The resulting $U$-band light curve is displayed in the bottom panel of Fig. 2 .

The data analysis was performed on the filtered data and it was carried out by prewhitening successive frequency peaks in the LNP until no other frequency was apparent. The analysis was applied independently to the $U_{-}, B$ - and $V$-band light curves. In this way we were able to identify four frequencies. We illustrate our findings in Fig. 3 with the $U$-band data. 
Table 3 shows for each wave band the value of the four identified frequencies and associated amplitudes. The data set length does not allow accurate phase determination. As a result we do not discuss it further.

Figure 2 shows the filtered light curve for the three observed wave bands, with the best fit that results from the four frequencies superimposed as solid lines.

The $\mathrm{f} 1$ and $\mathrm{f} 2$ frequencies are in good agreement (within the uncertainties) to those revealed by the December 2000 observations. Given the better quality of the 2001 data the uncertainties are now smaller. Additionally, we tentatively identify a third and a fourth frequencies. The current data do not allow to unequivocally demonstrate their presence. In any case, the phase diagrams hint at their presence.

\section{Discussion}

The Herbig Ae star V346 Ori is found to oscillate on timescales typical of $\delta$-Scuti stars, as predicted by its location near the PMS instability strip. Two frequencies are clearly identified. Whether other frequencies are present in the star's light curve is still open to debate. We hint at the presence of at least two other frequencies but more data are needed in order to be conclusive.

We follow the same approach as adopted by Marconi et al. (2001) in trying to reproduce the observed $\mathrm{f} 1$ and $\mathrm{f} 2$ frequencies by means of linear non-adiabatic radial oscillation models for PMS stars. We find that these frequencies are well reproduced for a PMS star of $1.55 \pm 0.15 M_{\odot}$, with $T_{\text {eff }}=$ $7410 \pm 150 \mathrm{~K}$ and $\log L / L_{\odot}=0.74 \pm 0.05$ pulsating in the fundamental (f2) and in the second overtone (f1). This solution is plotted in the HR diagram of Fig. 4, as a filled circle. The 1.5 $M_{\odot}$ PMS evolutionary track intersects the V346 Ori position inside the PMS instability strip, very near the ZAMS. We note that given our assumption of radial oscillations, mode identification is crucial if one is to be conclusive regarding the global parameters of the star.

The proposed result for V346 Ori's effective temperature is in good agreement with the Valenti et al. (2000) revised estimate for the spectral type of V346 Ori. Based on International Ultraviolet Explorer spectra, these authors propose that V346 Ori is A8 rather than A5 (van den Ancker et al. 1998). On the other hand, the new luminosity estimate implies that this star is further away than the Hipparcos lowest distance estimate (van den Ancker et al. 1998; lower triangle in Fig. 4), but still significantly closer than the Orion star forming region (upper triangle). This impinges directly on the interpretation of V346 Ori as a PMS object. In fact, based on the star's position in the surface gravity vs. effective temperature diagram, Kovalchuk \& Pugach (1997) claim that V346 Ori is not a PMS star. Just from the star's location on the HR diagram it is impossible to tell whether V346 Ori is a PMS star. With the current data we are not in a position to fully address V346 Ori's evolutionary state. A way to resolve this problem is by doing asteroseismology of non-radial modes (eg. Suran et al. 2001). For this, observations with better time sampling and spanning a longer time scale are needed.

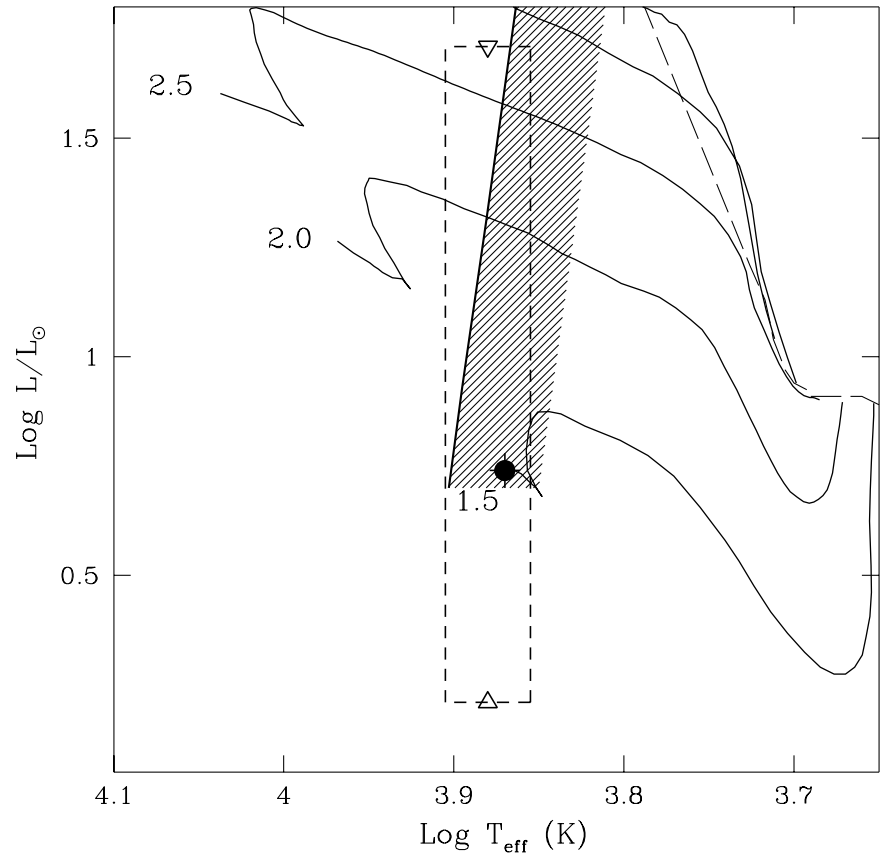

Fig. 4. Position of V346 in the H-R diagram using the first two frequencies (circle). Shaded region: PMS instability strip; dashed box: empirical uncertainty on distance and spectral type of V346 Ori; solid lines: PMS evolutionary tracks for 1.5, 2.0 and $2.5 M_{\odot}$ (from Palla \& Stahler 1993).

Acknowledgements. We would like to thank the staff of the Loiano Observatory for their kind support. FJGP and MJPFGM acknowledges support from project ESO/FNU/43658/2001. DFMF acknowledges financial support by FCT from the "Subprograma Ciência e Tecnologia do $3^{\circ}$ Quadro Comunitário de Apoio" and also from project POCTI/1999/FIS/34549, approved by FCT and POCTI, with funds from the European Union programme FEDER.

\section{References}

Covino, E., Melo, C., Alcalá, J. M., et al. 2001, A\&A, 375, 130 Hillenbrand, L. 1997, AJ, 113, 1733

Kurtz, D. W., \& Catala, C. 2001, A\&A, 369, 981

Kovalchuk, G. U., \& Pugach, A. E. 1997, A\&A, 325, 1077

Kurtz, D. W., \& Marang, F. 1995, MNRAS, 276, 191

Lomb, N. R. 1976, Ap\&SS, 39, 447

Marconi, M., \& Palla, F. 1998, ApJ, 507, L141

Marconi, M., Ripepi, V, Alcalá , J. M., et al. 2000, A\&A, 355, L35

Marconi, M., Ripepi, V, Bernabei, S., et al. 2001, A\&A, 372, L21

Marconi, M., Palla, F., \& Ripepi, V. 2002, Comm. in Asteroseismology, 141, 13

Palla, F., \& Stahler, S. 1993, ApJ, 418, 414

Palla, F., \& Stahler, S. 2001, ApJ, 553, 299

Popper, D. 1980, ARA\&A, 18, 115

Scargle, J. D. 1982, ApJ, 263, 835

Suran, M., Goupil, M., Baglin, A., Lebreton, Y., \& Catala, C. 2001, A\&A, 372, 233

Thé, P. S., de Winter, D., \& Pérez, M. R. 1994, A\&A, 104, 315

Valenti, J. A., Johns-Krull, C. M., \& Linsky, J. L. 2000, ApJS, 129, 399

van den Ancker, M., de Winter, D., \& Tjin a Djie, H. 1998, A\&A, 330, 145 